\title{
Photoelectron spectra of alkali metal-ammonia microjets: From blue electrolyte to bronze metal
}

\section{$\operatorname{AUTHOR}(S)$ :}

Buttersack, Tillmann; Mason, Philip E.; McMullen, Ryan S.; Schewe, H. Christian; Martinek, Tomas; Brezina, Krystof; Crhan, Martin; ... Winter, Bernd; Bradforth, Stephen E.; Jungwirth, Pavel

\section{CITATION:}

Buttersack, Tillmann ...[et al]. Photoelectron spectra of alkali metal-ammonia microjets: From blue electrolyte to bronze metal. Science 2020, 368(6495): 1086-1091

\section{ISSUE DATE:}

2020-06-05

URL:

http://hdl.handle.net/2433/254491

\section{RIGHT:}

This is the author's version of the work. It is posted here by permission of the AAAS for personal use, not for redistribution. The definitive version was published in Science Vol. 368, Issue 6495, pp. 1086-1091, 05 Jun 2020, DOl: 10.1126/science.aaz7607.; この論文は出版社版でありません。引用の際には出版社版をご確認ご利用ください。;This is not the published version. Please cite only the published version. 


\section{Photoelectron spectra of alkali metal-ammonia microjets: From blue electrolyte to bronze metal}

Tillmann Buttersack, ${ }^{\dagger 1,2}$ Philip E. Mason, ${ }^{\dagger 1}$ Ryan S. McMullen, ${ }^{\dagger 2}$, H. Christian Schewe, ${ }^{\dagger 3}$ Tomas Martinek, ${ }^{1}$ Krystof Brezina, ${ }^{1,4}$ Martin Crhan, ${ }^{1}$ Axel Gomez, ${ }^{1,5}$ Dennis Hein, ${ }^{6,7}$ Garlef Wartner, ${ }^{6,7}$ Robert Seidel, ${ }^{6,7}$ Hebatallah Ali, ${ }^{3}$ Stephan Thürmer, ${ }^{8}$ Ondrej Marsalek, ${ }^{* 4}$ Bernd Winter, ${ }^{* 3}$ Stephen E. Bradforth, ${ }^{* 2}$ and Pavel Jungwirth ${ }^{1 *}$

${ }^{1}$ Institute of Organic Chemistry and Biochemistry, Czech Academy of Sciences, Flemingovo nám. 2, 16610 Prague 6, Czech Republic

${ }^{2}$ Department of Chemistry, University of Southern California, Los Angeles, CA 90089-0482, USA

${ }^{3}$ Molecular Physics, Fritz-Haber-Institut der Max-Planck-Gesellschaft, D-14195 Berlin, Germany

${ }^{4}$ Charles University, Faculty of Mathematics and Physics, Ke Karlovu 3, 12116 Prague 2, Czech Republic

${ }^{5}$ Departement de Chimie, École Normale Supérieure, PSL University, 75005 Paris, France ${ }^{6}$ Helmholtz-Zentrum Berlin für Materialien und Energie, D-14109 Berlin, Germany

${ }^{7}$ Humboldt-Universität zu Berlin, Department of Chemistry, Brook-Taylor-Str. 2, D-12489 Berlin, Germany

${ }^{8}$ Department of Chemistry, Graduate School of Science, Kyoto University, KitashirakawaOiwakecho, Sakyo-Ku, Kyoto 606-8502, Japan

*Correspondence to: ondrej.marsalek@mff.cuni.cz, winter@fhi-berlin.mpg.de, stephen.bradforth@usc.edu, and pavel.jungwirth@uochb.cas.cz

${ }^{\dagger}$ All four authors contributed equally. 
Abstract: Experimental studies of the electronic structure of excess electrons in liquids archetypal quantum solutes - have been largely restricted to very dilute electron concentrations. Here, we overcome this limitation by applying soft x-ray photoelectron spectroscopy to characterize excess electrons originating from steadily increasing amounts of alkali metals dissolved in refrigerated liquid ammonia microjets. As concentration rises, a narrow peak at $\sim 2$ $\mathrm{eV}$, corresponding to vertical photodetachment of localized solvated electrons and dielectrons, transforms continuously into a band with a sharp Fermi edge accompanied by a plasmon peak, characteristic of delocalized metallic electrons. In combination with ab initio calculations of localized electrons and dielectrons, we obtain a clear picture of the energetics and density of states of the ammoniated electrons over the gradual transition from dilute blue electrolytes to concentrated bronze metallic solutions.

One Sentence Summary: Photoelectron spectroscopy maps electrons in alkali metal - liquid ammonia microjets capturing the blue electrolyte-to-bronze colored metal transition. 
Since the discovery of spectacularly colored alkali metal - ammonia solutions in the early $19^{\text {th }}$ century, excess ammoniated electrons have attracted considerable attention, as reviewed recently by Zurek et al. (1) (see Thompson's classic monograph(2) for an overview of the older literature). Alkali metals are soluble in liquid ammonia up to concentrations reaching over 20 mol\% metal (MPM), i.e. one metal atom per about four solvent molecules(1). A transition from a blue electrolyte into a bronze/gold colored metallic solution upon increasing alkali metal concentration is accompanied by a liquid-liquid phase separation at low enough temperatures(1-7). The nature of the metallic transition both in liquid and crystalline alkali metal - ammonia systems, directly evidenced by orders of magnitude increase in electrical conductivity, has puzzled researchers for decades $(1,8-10)$ and is not yet understood in molecular detail. The chemical species involved include dilute solvated electrons and dielectrons as well as their various complexes with alkali metal cations(1) - all gradually coalescing into delocalized structures giving rise to a conduction band. A series of conferences, the so-called Colloques Weyl, was organized around this topic in the second half of the last century, resulting in a flurry of articles focusing primarily on the structure, thermodynamics, and electrical and magnetic properties of the alkali metal - ammonia solutions(11-16). Electrons in liquid ammonia have also been thoroughly studied with NMR and ESR techniques. The latter show a narrow structureless spin resonance line with $g$ value characteristic of a free electron spin, which broadens upon increasing the alkali metal concentration(2,17). Shifts in the ${ }^{1} \mathrm{H}$ and ${ }^{14} \mathrm{~N}$ NMR positions (Knight shifts) give a measure of the unpaired electron spin density at all constituent nuclei within the orbit of the molecules solvating the unpaired electron $(17,18)$. Shkrob has argued(19) that the Knight parameters from ${ }^{14} \mathrm{~N}$ NMR, electron spin echo relaxation, and ESR linewidth data, can 
only be interpreted as a transfer of a significant fraction of the spin density to the nitrogen atoms in the first solvent sphere.

The principal means to explore electronic structure and thus the binding energies and density of states of excess ammoniated electrons is photoelectron spectroscopy (PES). Liquid ammonia has a great advantage over water in that high concentrations of ammoniated electrons can be reached in solutions that are stable for extended periods of time without the danger of explosion(20). Nevertheless, compared to the number of such investigations of electrons solvated in water (i.e., hydrated electrons)(21-23), PES studies in liquid ammonia are scarce. Early photoelectron total emission yield experiments led to an estimate of the photoelectron threshold of about $1.4 \mathrm{eV}(24,25)$, in good agreements with electrochemical determination of the adiabatic binding energy of an ammoniated electron(26). This value is also roughly consistent with results from cluster extrapolations(27-31). However, clusters have only limited relevance to the liquid bulk systems, as they inevitably exhibit large surface effects and are typically solid rather than liquid(32, 33); as a result, e.g., metastable cluster structures exist, characterized by low electron binding energies, which have no liquid bulk analogue(33), and also electron scattering from clusters differs from condensed phase data(34). Additional insight into the ultrafast dynamics of ammoniated electrons emerged from femtosecond time-resolved experiments involving multiphoton photoionization in pure liquid ammonia or photoexcitation in dilute alkali metalammonia solutions(35-38). These studies have typically probed ammoniated electrons in the low concentration regime, i.e., individual electrons well below the electrolyte-to-metal transition. In concentrated systems, plasmons in metallic lithium-ammonia solutions were explored by x-ray scattering two decades ago(10) and a PES study of small to medium-sized cryogenic sodiumammonia clusters was performed recently(31). 
There is thus a clear need for a direct PES investigation of excess ammoniated electrons that would cover both the electrolyte and metallic regimes. Recently, we have overcome a critical obstacle in collecting photoelectrons from a volatile polar refrigerated liquid. Namely, we developed an experimental setup that produces a liquid ammonia microjet and performed PES measurements with this new-generation apparatus(39). In that study, we characterized the valence and core orbital structure of pure gaseous and liquid ammonia and quantified the effect of the condensed phase environment on the orbital energies, which was found to be even stronger than in water, despite weaker hydrogen bonding in liquid ammonia (40). This work has paved the way for PES investigations of liquid alkali metal - ammonia solutions of increasing concentrations mapping the electrolyte-to-metal transition, as reported here.

Electronic structure calculations enable interpretation of PES measurements of ammoniated electrons in terms of a complex structural, dynamical, and molecular orbital picture. So far, only molecular pseudopotential calculations have been performed for electrons in liquid ammonia(41, 42), with density functional theory (DFT) applied to crystalline alkali metal ammonia systems(8). Although the early liquid state calculations provided some insight into the transition from individual solvated electrons through dielectrons (which exist as spin-paired singlet species in liquid ammonia(43)) to the onset of delocalized states upon increasing alkali metal loading, they were inevitably of a qualitative nature only. This was due both to neglect of the explicit electronic structure of the solvent and to approximations made in the pseudopotential itself(33). We have shown previously that in aqueous solutions a quantitative picture of the electronic structure of hydrated electrons and surrounding water molecules can be obtained using DFT-based ab initio molecular dynamics (AIMD)(44). Here we employ an extension of this 
approach combining it with quantum chemical embedded cluster evaluation of electron binding energies to characterize ammoniated electrons and dielectrons.

\section{Photoelectron spectroscopy: Electrolyte solutions}

Photoelectron (PE) experiments were carried out with the SOL ${ }^{3} \mathrm{PES}$ experimental setup(45) at the U49/2-PGM-1 beamline at the synchrotron radiation facility BESSY II(46) (for details see Experimental Methodology and Supplementary Material (SM)).PE spectra at low electron binding energies of microjets of lithium - liquid ammonia solutions at alkali metal concentrations ranging from 0.012 to 9.7 MPM are presented in Figure 1A. Analogous lowenergy spectra of potassium - liquid ammonia solutions (0.15 - 1.25 MPM) and sodium - liquid ammonia (0.15 - 0.75 MPM) are shown in Figures 1B and 1C. Visually, the increase of alkali metal concentration is connected with deepening of the characteristic blue color of the solutions, with the higher concentrations becoming practically black even in the thin microjet and the solution with the highest lithium concentration acquiring a discernible bronze colored metallic sheen.

Similarly to previously studied aqueous microjets $(47,48)$, in experiments with liquid ammonia we also observe electrostatic effects leading to global PE spectral shifts. These shifts are larger for alkali metals in liquid ammonia than for solutions of alkali halide salts at equivalent concentrations(49). To correct for these instrumental spectral shifts, the low concentration spectra (0.08 MPM for Li and 0.15 MPM for Na and K) were aligned horizontally such that the lowest-energy liquid ammonia peak (3a1), fitted to a Gaussian, was always anchored at $9.09 \mathrm{eV}$, which is the value of the corresponding vertical detachment energy (VDE) as determined in our recent PE measurements of a pure liquid ammonia microjet(39). All the other spectra are aligned using the exact same shift. This procedure (see SM for more details) is 


\section{Sclence Submitted Manuscript: Confidential}

\section{DIAAAS}

well justified as for low to moderate alkali metal concentrations the effect of ionic solutes on the position of the solvent PE peaks has been found to be negligible in water (see below and Refs. $(47,48))$. Nevertheless, these factors add together to produce a small systematic uncertainty in determining absolute values of VDEs, which we estimate not to exceed $\sim 0.4 \mathrm{eV}$.

A substantial result of the present measurements is that from about 0.08 to 1 MPM the PE spectra consistently show a small, but clearly visible peak at a VDE of about 2 eV (Figure 1). The integrated area of this peak is roughly linearly proportional to the number concentration of the alkali metal (Figure 1D). The observation that the position of this peak practically does not depend on the chemical nature of the alkali metal points directly to ammoniated electrons. More precisely, starting from $\sim 10^{-3}$ MPM the solvated electrons engage in spin-pairing forming dielectrons $(1,2,50)$. Electron spin resonance measurements provide an estimate of the concentration dependence of the dielectron/electron ratio(2), which increases with dissolved metal concentration and at around 0.1 MPM reaches a factor of $\sim 10$. The measured value of $\sim 2$ $\mathrm{eV}$ thus corresponds primarily to the VDE of dielectrons.

\section{Electronic structure calculations}

Our experimental conclusions are further supported by electronic structure calculations. In order to faithfully model the structure of electrons, dielectrons, as well as electron - alkali cation pairs (see Figure S10 in the SM) in liquid ammonia we need to go beyond static ab initio calculations of small clusters on the one hand(51) and molecular pseudopotential bulk simulations on the other hand(41-43). Here, we combine state-of-the-art AIMD using the revPBE0-D3 hybrid density functional for sampling of relevant structures with subsequent second order Möller-Plesset perturbation theory (MP2) for VDE calculations. The latter 
calculations are performed for clusters carved out of the AIMD trajectory and embedded in a polarizable continuum model (PCM). See SM for details.

AIMD simulations of an excess electron in bulk liquid ammonia demonstrate that an ammoniated electron occupies a cavity coordinated by roughly 12 ammonia molecules and has a gyration radius of $3.9 \AA$ on average (Figure $2 \mathrm{~A}$ ), consistent with the value of $\sim 3.5 \AA$ following from a moment analysis of the optical absorption spectra(52). The spin-paired ammoniated dielectron adopts a similar structure to the electron (Figure 2B) with about the same number of ammonia molecules in contact and a slightly larger average gyration radius of $4.4 \AA$. In both cases, the solvent shell is very diffuse and lacks clear separation from the rest of the solvent. Our test AIMD calculations show that adding a second electron of the same spin leads to the formation of two separate solvated electron cavities, rather than a dielectron in a single cavity.

The electron solvation structure in ammonia is qualitatively similar but quantitatively different from that of a hydrated electron in water or aqueous solutions(33, 44). Namely, the first solvent shell of the hydrated electron is significantly more structured and less diffuse compared to those of the ammoniated electron or dielectron. Moreover, the hydrated electron is much smaller, with only 4 to 6 water molecules in its hydration shell and a gyration radius of $\sim 2.5$ $\AA(44,52)$. Concerning dielectrons, the situation in liquid ammonia is likely to be different from that in water, where hydrated dielectrons are predicted to be thermodynamically substantially less stable than hydrated electrons(53, 54).

The AIMD simulations also serve as a basis for calculations of the VDE of the ammoniated electron and dielectron. First, we carved out the immediate electron solvation shells containing $12 \mathrm{NH}_{3}$ molecules from more than hundred snapshots from the AIMD trajectories. These structures were then embedded in a polarizable continuum model with the dielectric 


\section{Science}

Submitted Manuscript: Confidential

\section{MIAAS}

constant of liquid ammonia (see below and the SM for more details). The distributions of the resulting VDEs of the two species evaluated at the MP2 level (without any additional shifts or adjustments) are plotted in Figure 3, referenced against our experimental data. The calculated distributions have width of about $0.3 \mathrm{eV}$ peaking at around $2.0 \mathrm{eV}$ for the ammoniated electron and $\sim 1.6 \mathrm{eV}$ for the dielectron. Comparing to our low-concentration experimental spectra we see that the experimental peak at $\sim 2 \mathrm{eV}$ encompasses within its width both the calculated solvated electron and dielectron VDE distributions (Figure 3). These results are consistent with the previously calculated very small difference of $\sim 0.1 \mathrm{eV}$ between the lowest optical transitions of an ammoniated electron and spin-paired dielectron in a rather idealized six-coordinated cluster geometry(55). The value for the ammoniated electron, however, differs quantitatively from extrapolations from ammonia clusters with an excess electron(29) yielding $1.25 \mathrm{eV}$. This is due to the fact that the cryogenic clusters are finite and solid and, therefore, have different properties from those of the bulk liquid systems investigated here(56, 57).

\section{Photoelectron spectroscopy: From electrolytes to metallic solutions}

Upon increasing the alkali metal concentration, the PE spectra exhibit a gradual conversion of the Gaussian-type solvated electron peak into an asymmetric band with a sharp edge toward lower binding energies accompanied by one or two satellite peaks on the higher binding energy side (Figures 1 and 4 with details provided in the SM). At the lowest alkali metal concentrations, the solvated electron peak can be fitted to a Gaussian (Figure 4) with a full width at half maximum of about 0.45 to $0.6 \mathrm{eV}$ (i.e., slightly narrower than the equivalent first ionization peak for halides in liquid ammonia(49)) and with a low energy onset (appearance 


\section{DIAAAS}

potential) at around $1.5 \mathrm{eV}$, which is close to the previous estimate of $1.4 \mathrm{eV}$ from the photoelectron threshold measurements(24, 25).

In contrast, at the highest lithium concentration of 9.7 MPM the PE spectrum is fitted to an inverse-parabola conduction band with a sharp Fermi edge and two plasmon peaks (Figure 4), as follows directly from the free electron gas model for metals(58), with an effective electron mass close to unity (Figure 4 and Table 1). Due to the relatively low electron density the plasmon frequency is in the visible range which gives the concentrated alkali metal - ammonia solutions their characteristic bronze/gold color(1, 59). A conduction band with a Fermi edge and a plasmon peak can also be observed for the 1.25 MPM potassium - ammonia solution, whereas for sodium - ammonia we could not prepare homogeneous solutions above 1 MPM due to spontaneous phase separation at the experimental conditions(1, 2).

An analogous fit to a free electron gas model is shown for a microjet PE spectrum of liquid 50:50 sodium - potassium metal alloy (Figure 4A and Table 1). Here, the conduction band is wider and the fundamental plasmon excitation seen at higher binding energies has a higher frequency $(\sim 4.5 \mathrm{eV})$ as expected for the higher electron density in the metal alloy compared to the metallic lithium - ammonia solutions. This higher frequency places the plasmon in the UV when considering the optical reflectance of the sodium - potassium alloy, which does not exhibit any color and has a metallic silver sheen. This is actually true for all alkali metals except for cesium, in which the lower free electron density shifts the plasmon frequency to the visible range conferring a golden color(59).

The PE spectra of the 1 - 4 MPM lithium - liquid ammonia solutions can be fitted by a combination of a localized Gaussian at $2 \mathrm{eV}$ with a conduction band and plasmon following from a free electron gas model, albeit with a reduced effective electron mass (Figure 4D and Table 1). 
As the lithium concentration increases, the relative weight of the Gaussian contribution to the spectrum decreases such that at 9.7 MPM it practically vanishes (Figure 4E). At the same time, the spectra exhibit changes in the shape and position of the liquid ammonia 3a1 peak upon buildup of the metallic behavior of the solution (Figure 4C). Namely, in the electrolyte regime the position of the 3a1 peak almost does not change, but it does tend to broaden and move to lower binding energies upon appearance of the metallic state (for more details see the SM).

Table 1: Key parameters for lithium-ammonia solutions and the sodium - potassium alloy: concentrations in MPM, electron densities $n$, effective electron masses $m_{e}{ }^{*}$, widths of the conduction band $E_{c}$, and the positions of the plasmon peak $E_{p}$ with respect to the Fermi energy $E_{F}$. The latter two were gained from fitting to a free electron gas model with the above effective electron masses. The effective electron masses $m_{e}{ }^{*}$ were obtained by fitting described in SM.

\begin{tabular}{|c|c|c|c|c|c|c|c|}
\hline$c(\mathrm{MPM})$ & $\begin{array}{r}\mathrm{NaK} \\
100\end{array}$ & $\begin{array}{c}\mathrm{Li} \mathrm{NH}_{3} \\
9.7\end{array}$ & $\begin{array}{c}\mathrm{Li@NH} 3 \\
3.4\end{array}$ & $\begin{array}{c}\mathrm{Li@NH} 3 \\
0.97\end{array}$ & $\begin{array}{c}\mathrm{Li@NH} 3 \\
0.35\end{array}$ & $\begin{array}{c}\mathrm{Li@NH} 3 \\
0.08\end{array}$ & $\begin{array}{c}{\mathrm{Li} @ N_{3}} \\
0.012\end{array}$ \\
\hline$c(\mathrm{M})$ & 29 & 4.3 & 1.4 & 0.39 & 0.14 & 0.03 & 0.005 \\
\hline $\mathrm{n}_{\mathrm{e}}\left(10^{21} \mathrm{~cm}^{-3}\right)$ & 16.3 & 2.15 & 1.05 & 0.25 & 0.09 & 0.02 & 0.003 \\
\hline $\mathrm{m}_{\mathrm{e}} * / \mathrm{m}_{\mathrm{e}}$ & 1.21 & 0.72 & 0.49 & 0.25 & --- & --- & --- \\
\hline $\mathrm{E}_{\mathrm{c}}(\mathrm{eV})$ & 2.06 & 0.85 & 0.74 & 0.98 & --- & --- & --- \\
\hline $\mathrm{E}_{\mathrm{p}}(\mathrm{eV})$ & 4.52 & 2.03 & 1.67 & 1.74 & --- & --- & --- \\
\hline
\end{tabular}

The above results suggest that, in accord with the previous view highlighted in Ref. (1), the electrolyte-to-metal transition upon increasing the metal concentration in alkali metal ammonia solutions is not a sharp phase transition, but rather a gradual conversion resembling a percolation process, with an unresolved question concerning the sizes of potentially coexisting 
microscopic regions supporting localized and delocalized electrons $(2,35,60,61)$. This is a different picture than that of a sharp transition at 8 MPM drawn from recent PE spectra of alkali metal - ammonia nanodroplets(31). Although such experiments are pioneering in their own right, it is reasonable to question whether finite size clusters are representative of bulk metallic solutions in their electronic structure. There are actually significant differences observed for the cluster PE spectra, for example the lack of a sharp Fermi edge, the absence of plasmon peaks, and the Fermi edge onset appearing at higher rather than lower binding energies from the onset of the localized (di)electron peak(31). All of these factors suggest a qualitatively different transition in the nanodroplets, taking place at significantly higher concentrations, than determined for the bulk liquid systems previously(1, 31). Our present bulk liquid PES results show a build-up of a conduction band with a Fermi edge with increasing alkali metal concentration even before the solution becomes visibly metallic (Figure 4). This picture is also in accord with the semi-quantitative Mott's criterion postulating that a metallic state starts appearing when the mean distance between the electrons drops below approximately four times their size(62). With the radius of gyration of the ammoniated electrons and dielectrons of $\sim 4 \AA$ (Figure 2) metallic behavior should thus start evolving at around 1 MPM, which is consistent with the onset of conduction band formation in the present PES measurements. Note, however, that the transition observed here is more gradual than what would strictly follow from a pure Mott’s transition(62).

We can thus conclude that the occurrence in the PE spectrum of a conduction band with a distinct Fermi edge, together with plasmon peaks, is a signature of the electrolyte-to-metal transition. This gradual transition is observed both in the lithium - ammonia and potassium ammonia solutions, see Figures 1 and 4 and SM. (As mentioned above, sodium - ammonia 


\section{DIAAAS}

solutions phase-separate at concentrations above 1.5 MPM into immiscible electrolyte and metallic phases(2), which compromises the microjet PE measurements.) One can also view the process from the other side, i.e., as a metal-to-electrolyte transition upon decreasing the alkali metal concentration. We see from Figure 4 that at the highest studied concentration of 9.7 MPM the metallic lithium - ammonia system behaves very close to an ideal free electron gas, just as the liquid sodium - potassium alloy (similarly to pure alkali metals $(63,64)$ ). However, upon decreasing the concentration of the alkali metal - ammonia solutions below 4 MPM we observe departure from the ideal electron gas model, as exemplified by a rapid decrease of the effective electron mass well below the value of 1 me (Figure 4D). A schematized picture is presented in Figure 5 capturing the gist of the transition. It graphically depicts the gradual interconversion between localized “chemical” species - solvated electrons and dielectrons - into delocalized “physical” moieties, i.e., metallic conduction band electrons, upon changing the electron concentration.

\section{Outlook}

The present study shows that the electrolyte-to-metal transition in increasingly concentrated alkali metal - liquid ammonia solutions is a gradual process rather than an abrupt first order transition, which is in line with previous suggestions(1). From the molecular point of view, this transition may be understood in a simplified way as gradual coalescence of individual solvated electrons and dielectrons upon increasing alkali metal doping, with the metallic behavior appearing at around the percolation threshold. 
After overcoming methodological difficulties connected with modeling of the onset of the metallic state, future AIMD simulations of concentrated alkali metal - liquid ammonia solutions will shed more light on the electrolyte-to-metal transition in terms of the details of the underlying electronic structure and molecular geometries. On the experimental side, the experience already gained from liquid ammonia microjets is proving essential in our current attempts to realize the metallic state in the much more reactive (even explosive) alkali metal water systems.

\section{References and Notes:}

1. E. Zurek, P. P. Edwards, R. Hoffmann, A Molecular Perspective on Lithium-Ammonia Solutions. Angewandte Chemie-International Edition 48, 8198-8232 (2009).

2. J. C. Thompson, Electrons in Liquid Ammonia. (Clarendon Press, Oxford, 1976).

3. M. Lodge, P. Cullen, N. H. Rees, N. Spencer, K. Maeda, J. R. Harmer, M. O. Jones, P. P. Edwards, Multielement NMR Studies of the Liquid-Liquid Phase Separation and the Metal-to-Nonmetal Transition in Fluid Lithium- and Sodium-Ammonia Solutions. Journal of Physical Chemistry B 117, 13322-13334 (2013).

4. G. N. Chuev, P. Quemerais, Nature of meta-nonmetal transition in metal-ammonia solutions. II. From uniform metallic state to inhomogeneous electronic microstructure. Journal of Chemical Physics 128, 144503 (2008).

5. G. N. Chuev, P. Quemerais, J. Crain, Nature of the metal-nonmetal transition in metalammonia solutions. I. Solvated electrons at low metal concentrations. Journal of Chemical Physics 127, 244501 (2007). 
6. S. Hayama, N. T. Skipper, J. C. Wasse, H. Thompson, X-ray diffraction studies of solutions of lithium in ammonia: The structure of the metal-nonmetal transition. Journal of Chemical Physics 116, 2991-2996 (2002).

7. H. Thompson, J. C. Wasse, N. T. Skipper, S. Hayama, D. T. Bowron, A. K. Soper, Structural studies of ammonia and metallic lithium-ammonia solutions. Journal of the American Chemical Society 125, 2572-2581 (2003).

8. J. Kohanoff, F. Buda, M. Parrinello, M. L. Klein, Nature of the Conduction States in the Metallic Molecular Crystal Li(NH3)4. Physical Review Letters 73, 3133 (1994).

9. T. A. Kaplan, J. F. Harrison, J. L. Dye, R. Rencsok, Relation of Li(NH3)4 to Electrides. Physical Review Letters 75, 978 (1995).

10. C. A. Burns, E. D. Isaacs, P. Abbamonte, P. M. Platzman, Electronic excitations in lithium ammonia. Journal of Physics and Chemistry of Solids 61, 411-413 (2000).

11. Lepoutre G., Sienko M. J., Eds., Solutions Metal-Ammoniac: Proprietes PhysicoChimiques, (Benjamin, New York, 1964).

12. Lagowski J. J., Sienko M. J., Eds., Metal-Ammonia Solutions, (Butterworths, London, 1970).

13. Jortner J., Kestner N. R., Eds., Electrons in Fluids, (Springer, Heidelberg, 1973).

14. Webster B. (ed.), Electrons in Fluids - The Nature of Metal-Ammonia Solutions. Journal of Phsical Chemistry 84, 1065-1298 (1980).

15. Lepoutre G. (ed.), Excess Electrons and Metal-Ammonia Solutions. 88, 3699-3914 (1984).

16. Damay P. (ed.), Metals in Solution. Journal de Physique IV 1, C5 3-373 (1991). 
17. P. P. Edwards, Magnetic-Resonance Studies of Alikali-Metals in Nonaqueous Solvents. Journal of Physical Chemistry 88, 3772-3780 (1984).

18. M. C. R. Symons, Solutions of Metals - Solvated Electrons Chemical Society Reviews 5, 337-358 (1976).

19. I. A. Shkrob, Ammoniated electron as a solvent stabilized multimer radical anion. Journal of Physical Chemistry A 110, 3967-3976 (2006).

20. P. E. Mason, F. Uhlig, V. Vanek, T. Buttersack, S. Bauerecker, P. Jungwirth, Coulomb explosion during the early stages of the reaction of alkali metals with water. Nature Chemistry 7, 250-254 (2015).

21. D. M. Neumark, Ejecting electrons from water. Nature Chemistry 2, 247-248 (2010).

22. Y. Tang, H. Shen, K. Sekiguchi, N. Kurahashi, T. Mizuno, Y. I. Suzuki, T. Suzuki, Direct measurement of vertical binding energy of a hydrated electron. Physical Chemistry Chemical Physics 12, 3653-3655 (2010).

23. F. Buchner, T. Schultz, A. Lubcke, Solvated electrons at the water-air interface: surface versus bulk signal in low kinetic energy photoelectron spectroscopy. Physical Chemistry Chemical Physics 14, 5837-5842 (2012).

24. J. Häsing, Über die lichtelektrischen Eigenschaften der Lösungen von Natrium in flüssigem Ammoniak. Annalen der Physik 429, 509-533 (1940).

25. H. Aulich, B. Baron, P. Delahay, R. Lugo, Photoelectron Emission by Solvated Electrons in Liquid-Ammonia Journal of Chemical Physics 58, 4439-4443 (1973).

26. T. Teherani, K. Itaya, A. J. Bard, Electrochemical Study of Solvated Electrons in LiquidAmmonia. Nouveau Journal De Chimie-New Journal of Chemistry 2, 481-487 (1978). 
27. H. Haberland, C. Ludewigt, H. G. Schindler, D. R. Worsnop, Clusters of Water and Ammonia with Excess Electrons Surface Science 156, 157-164 (1985).

28. R. Takasu, F. Misaizu, K. Hashimoto, K. Fuke, Microscopic solvation process of alkali atoms in finite clusters: Photoelectron and photoionization studies of M(NH3)(n) and M(H2O)(n) (M=Li, Li-, Na-). Journal of Physical Chemistry A 101, 3078-3087 (1997).

29. H. W. Sarkas, S. T. Arnold, J. G. Eaton, G. H. Lee, K. H. Bowen, Ammonia cluster anions and their relationship to ammoniated (solvated) electrons: The photoelectron spectra of (NH3)(-)(n=41-1100). Journal of Chemical Physics 116, 5731-5737 (2002).

30. T. Zeuch, U. Buck, Sodium doped hydrogen bonded clusters: Solvated electrons and size selection. Chemical Physics Letters 579, 1-10 (2013).

31. S. Hartweg, A. H. C. West, B. L. Yoder, R. Signorell, Metal Transition in SodiumAmmonia Nanodroplets. Angewandte Chemie-International Edition 55, 12347-12350 (2016).

32. O. Marsalek, F. Uhlig, P. Jungwirth, Electrons in Cold Water Clusters: An ab Initio Molecular Dynamics Study of Localization and Metastable States. Journal of Physical Chemistry C 114, 20489-20495 (2010).

33. O. Marsalek, F. Uhlig, J. Vandevondele, P. Jungwirth, Structure, Dynamics, and Reactivity of Hydrated Electrons by Ab Initio Molecular Dynamics. Accounts of Chemical Research 45, 23-32 (2012).

34. T. E. Gartmann, S. Hartweg, L. Ban, E. Chasovskikh, B. L. Yoder, R. Signorell, Electron scattering in large water clusters from photoelectron imaging with high harmonic radiation. Physical Chemistry Chemical Physics 20, 16364-16371 (2018). 
35. P. Vöhringer, in Annual Review of Physical Chemistry, Vol 66, M. A. Johnson, T. J. Martinez, Eds. (2015), vol. 66, pp. 97-118.

36. T. Vogler, P. Vöhringer, Probing the band gap of liquid ammonia with femtosecond multiphoton ionization spectroscopy. Physical Chemistry Chemical Physics 20, 2565725665 (2018).

37. J. Lindner, A.-N. Unterreiner, P. Vöhringer, Femtosecond relaxation dynamics of solvated electrons in liquid ammonia. ChemPhysChem 7, 363-369 (2006).

38. J. Lindner, A.-N. Unterreiner, P. Vöhringer, Femtosecond spectroscopy of solvated electrons from sodium-ammonia-d3 solutions: temperature jump versus local density jump. Journal of Chemical Physics 129, 064514 (2008).

39. T. Buttersack, P. E. Mason, R. S. McMullen, T. Martinek, K. Brezina, D. Hein, H. Ali, C. Kolbeck, C. Schewe, S. Malerz, B. Winter, R. Seidel, O. Marsalek, P. Jungwirth, S. E. Bradforth, Valence and Core-Level X-ray Photoelectron Spectroscopy of a Liquid Ammonia Microjet. Journal of the American Chemical Society 141, 1838-1841 (2019).

40. B. Winter, R. Weber, W. Widdra, M. Dittmar, M. Faubel, I. V. Hertel, Full valence band photoemission from liquid water using EUV synchrotron radiation. Journal of Physical Chemistry A 108, 2625-2632 (2004).

41. Z. H. Deng, G. J. Martyna, M. L. Klein, Electronic States in Metal-Ammonia Solutions Physical Review Letters 71, 267-270 (1993).

42. Z. H. Deng, G. J. Martyna, M. L. Klein, Quantum Simulation Studies of Metal-Ammonia Solutions Journal of Chemical Physics 100, 7590-7601 (1994).

43. G. J. Martyna, Z. H. Deng, M. L. Klein, Quantum simulation studies of singlet and triplet bipolarons in liquid ammonia. Journal of Chemical Physics 98, 555-563 (1992). 
44. F. Uhlig, O. Marsalek, J. P., Unraveling the Complex Nature of the Hydrated Electron. Jounal of Physical Chemistry Letters 3, 3071-3075 (2012).

45. R. Seidel, M. N. Pohl, H. Ali, B. Winter, E. F. Aziz, Advances in liquid phase soft-x-ray photoemission spectroscopy: A new experimental setup at BESSY II. Review of Scientific Instruments 88, (2017).

46. K. J. S. Sawhney, F. Senf, W. Gudat, PGM beamline with constant energy resolution mode for U49-2 undulator at BESSY-II. Nuclear Instruments \& Methods in Physics Research Section a-Accelerators Spectrometers Detectors and Associated Equipment 467, 466-469 (2001).

47. B. Winter, M. Faubel, Photoemission from liquid aqueous solutions. Chemical Reviews 106, 1176-1211 (2006).

48. M. Pohl, E. Muchova, R. Seidel, H. Ali, S. Srsen, I. Wilkinson, B. Winter, P. Slavicek, Do water's electrons care about electrolytes? Chemical Science 10, 848-865 (2019).

49. T. Buttersack, P. E. Mason, P. Jungwirth, C. Schewe, B. Winter, R. Seidel, R. McMullen, S. E. Bradforth, Deeply Cooled and Temperature Controlled Microjets: Liquid Ammonia Solutions Released into Vacuum for Analysis by Photoelectron Spectroscopy. Review of Scientific Instruments, DOI: 10.1063/1.5141359, (2020).

50. U. Schindewolf, M. Werner, Thermodynamics of Sodium-Ammonia Solutions. Journal of Physical Chemistry 84, 1123-1127 (1980).

51. T. Sommerfeld, K. M. Dreux, Characterizing the excess electron of Li(NH3)4. Journal of Chemical Physics 137, 244302 (2012).

52. S. Golden, T. R. Tuttle, Nature of Solvated Electron Absorption-Spectra Journal of the Chemical Society-Faraday Transactions Ii 75, 474-484 (1979). 
53. R. E. Larsen, B. J. Schwartz, Full Configuration Interaction Computer Simulation Study of the Thermodynamic and Kinetic Stability of Hydrated Dielectrons. Journal of Physical Chemistry B 110, 1006-1014 (2006).

54. K. H. Schmidt, D. M. Bartels, Lack of ionic strength effect in the recombination of hydrated electrons: (e -) aq + (e -) aq $\rightarrow 2(\mathrm{OH}-)+$ H 2. Chemical Physics 190, 145-152 (1995).

55. M. Mauksch, S. B. Tsogoeva, Spin-paired solvated electron couples in alkali-ammonia systems. Physical Chemistry Chemical Physics 20, 27740-27744 (2018).

56. O. Marsalek, F. Uhlig, T. Frigato, B. Schmidt, P. Jungwirth, Dynamics of Electron Localization in Warm versus Cold Water Clusters. Physical Review Letters 105, 043002 (2010).

57. L. Ma, K. Majer, F. Chirot, B. von Issendorff, Low temperature photoelectron spectra of water cluster anions. Journal of Chemical Physics 131, 144303 (2009).

58. C. Kittel, Introduction to Solid State Physics. (John Wiley \& Sons, Inc., Hoboken NJ, 2005).

59. C. C. Addison, The Chemistry of Liquid Alkali Metals. (John Wiley \& Sons, New York, 1984).

60. J. Jortner, M. H. Cohen, Metal-Nonmetal Transition in Metal-Ammonia Solutions Physical Review B 13, 1548-1568 (1976).

61. P. Damay, P. Chieux, Influence of Critical Fluctuations of Concentrations on the Transport Properties of Metal-Ammonia Solutions. Journal of Physical Chemistry 84, 1203-1205 (1980). 
62. P. P. Edwards, M. T. J. Lodge, F. Hensel, R. Redmer, ' ... a metal conducts and a nonmetal doesn't'. Philosophical Transactions of the Royal Society a-Mathematical Physical and Engineering Sciences 368, 941-965 (2010).

63. G. Indlekofer, P. Oelhafen, Electronic-Structure of Liquid Lithium and Sodium from Electron-Spectroscopy. Journal of Non-Crystalline Solids 117, 340-343 (1990).

64. G. Indlekofer, P. Oelhafen, The Electronic Structure of Liquid Heavy Alkali-Metals from Electron-Spectroscopy Journal of Non-Crystalline Solids 156, 226-231 (1993).

65. T. Buttersack, P. E. Mason, R. S. McMullen, H. C. Schewe,T. Martinek, K. Brezina, M. Crhan, A. Gomez, D. Hein, G. Wartner, R. Seidel, H. Ali, S. Thürmer, O. Marsalek, B. Winter, S. E. Bradforth, P. Jungwirth, Zenodo, DOI:10.5281/zenodo.3733932 (2020)

66. J. Hutter, M. Iannuzzi, F. Schiffmann, J. VandeVondele, CP2K: atomistic simulations of condensed matter systems. Wiley Interdisciplinary Reviews-Computational Molecular Science 4, 15-25 (2014).

67. J. VandeVondele, M. Krack, F. Mohamed, M. Parrinello, T. Chassaing, J. Hutter, QUICKSTEP: Fast and accurate density functional calculations using a mixed Gaussian and plane waves approach. Computer Physics Communications 167, 103-128 (2005).

68. B. Eckl, J. Vrabec, H. Hasse, An optimised molecular model for ammonia. Molecular Physics 106, 1039-1046 (2008).

69. Y. K. Zhang, W. T. Yang, Comment on "Generalized gradient approximation made simple". Physical Review Letters 80, 890-890 (1998).

70. S. Grimme, J. Antony, S. Ehrlich, H. Krieg, A consistent and accurate ab initio parametrization of density functional dispersion correction (DFT-D) for the 94 elements H-Pu. Journal of Chemical Physics 132, 154104 (2010). 
71. S. Goedecker, M. Teter, J. Hutter, Separable dual-space Gaussian pseudopotentials. Physical Review B 54, 1703-1710 (1996).

72. J. P. Perdew, K. Burke, M. Ernzerhof, Generalized gradient approximation made simple. Physical Review Letters 77, 3865-3868 (1996).

73. L. Goerigk, S. Grimme, A thorogh benchmark of density functional methods for general main group thermochemistry, kinetics, and noncovalents interactions, Physical Chemistry Chemical Physics, 13, 6670-6688 (2011).

74. M. Guidon, J. Hutter, J. VandeVondele, Auxiliary density matrix methods for HartreeFock exchange calculations, Journal of Chemical Theory and Computation 6, 2348-2364 (2010).

75. A. Ricci, G. Ciccotti, Algorithms for Brownian dynamics. Molecular Physics 101, 19271931 (2003).

76. G. Bussi, D. Donadio, M. Parrinello, Canonical sampling through velocity rescaling. Journal of Chemical Physics 126, 014101 (2007).

77. O. Marsalek, F. Uhlig, J. P., Electrons in Cold Water Clusters: An ab Initio Molecular Dynamics Study of Localization and Metastable States. Journal of Physical Chemistry 114, 20489-20495 (2010).

78. S. Miertus, E. Scrocco, J. Tomasi, Electrostatic Interaction of a Solute with a Continuum - A Direct Utilization of Ab Initio Molecular Potentials for the Prevision of Solvent Effects Chemical Physics 55, 117-129 (1981).

79. J. R. Rumble, Ed., CRC Handbook of Chemistry and Physics 100th Edition, (CRC Press Taylor \& Francis Group, 2019). 


\section{Sclence Submitted Manuscript: Confidential}

DIAAAS

80. E. Cances, B. Mennucci, J. Tomasi, A new integral equation formalism for the polarizable continuum model: Theoretical background and applications to isotropic and anisotropic dielectrics. Journal of Chemical Physics 107, 3032-3041 (1997).

81. M. Cossi, V. Barone, Solvent effect on vertical electronic transitions by the polarizable continuum model. Journal of Chemical Physics 112, 2427-2435 (2000).

82. M. J. T. Frisch, G. W.; Schlegel, H. B.; Scuseria, G. E.; Robb, M. A.; Cheeseman, J. R.; Scalmani, G.; Barone, V.; Mennucci, B.; Petersson, G. A.; Nakatsuji, H.; Caricato, M.; Li, X.; Hratchian, H. P.; Izmaylov, A. F.; Bloino, J.; Zheng, G.; Sonnenberg, J. L.; Hada, M.; Ehara, M.; Toyota, K.; Fukuda, R.; Hasegawa, J.; Ishida, M.; Nakajima, T.; Honda, Y.; Kitao, O.; Nakai, H.; Vreven, T.; Montgomery, Jr., J. A.; Peralta, J. E.; Ogliaro, F.; Bearpark, M.; Heyd, J. J.; Brothers, E.; Kudin, K. N.; Staroverov, V. N.; Kobayashi, R.; Normand, J.; Raghavachari, K.; Rendell, A.; Burant, J. C.; Iyengar, S. S.; Tomasi, J.; Cossi, M.; Rega, N.; Millam, J. M.; Klene, M.; Knox, J. E.; Cross, J. B.; Bakken, V.; Adamo, C.; Jaramillo, J.; Gomperts, R.; Stratmann, R. E.; Yazyev, O.; Austin, A. J.; Cammi, R.; Pomelli, C.; Ochterski, J. W.; Martin, R. L.; Morokuma, K.; Zakrzewski, V. G.; Voth, G. A.; Salvador, P.; Dannenberg, J. J.; Dapprich, S.; Daniels, A. D.; Farkas, Ö.; Foresman, J. B.; Ortiz, J. V.; Cioslowski, J.; Fox, D. J. . (Gaussian, Inc., Wallingford CT, 2016).

83. J. M. Herbert, M. Head-Gordon, Calculation of electron detachment energies for water cluster anions: An appraisal of electronic structure methods, with application to (H2O)(20)over-bar and (H2O)(24)over-bar. Journal of Physical Chemistry A 109, 52175229 (2005). 
84. J. D. Chai, M. Head-Gordon, Long-range corrected hybrid density functionals with damped atom-atom dispersion corrections. Physical Chemistry Chemical Physics 10, 6615-6620 (2008).

85. N. Kurahashi, S. Karashima, Y. Tang, T. Horio, B. Abulimiti, Y. I. Suzuki, Y. Ogi, M. Oura, T. Suzuki, Photoelectron spectroscopy of aqueous solutions: Streaming potentials of $\mathrm{NaX}(\mathrm{X}=\mathrm{Cl}, \mathrm{Br}$, and $\mathrm{I})$ solutions and electron binding energies of liquid water and $\mathrm{X}$. Journal of Chemical Physics 140, (2014).

86. N. Kurahashi, T. Suzuki, On the Relation between the Interfacial Charge of a Discharging Nozzle and Electrification of a Liquid Microjet. Chemistry Letters 47, 16-19 (2018).

87. S. Thürmer, R. Seidel, M. Faubel, W. Eberhardt, J. C. Hemminger, S. E. Bradforth, B. Winter, Photoelectron Angular Distributions from Liquid Water: Effects of Electron Scattering. Physical Review Letters 111, (2013).

88. N. Ottosson, M. Faubel, S. E. Bradforth, P. Jungwirth, B. Winter, Photoelectron spectroscopy of liquid water and aqueous solution: Electron effective attenuation lengths and emission-angle anisotropy. Journal of Electron Spectroscopy and Related Phenomena 177, 60-70 (2010).

89. G. Olivieri, A. Goel, A. Kleibert, D. Cvetko, M. A. Brown, Quantitative ionization energies and work functions of aqueous solutions. Physical Chemistry Chemical Physics 18, 29506-29515 (2016).

90. V. F. Hnizda, C. A. Kraus, Properties of Electrolyte Solutions 39. Conductance of Several Salts in Ammonia -34-Degrees by a Precision Method Journal of the American Chemical Society 71, 1565-1575 (1949). 


\section{Science \\ DIAAAS}

Submitted Manuscript: Confidential

91. J. L. Dye, R. F. Sankuer, G. E. Smith, Ion Transport in Sodium Ammonia Solutions.

Journal of the American Chemical Society 82, 4797-4803 (1960).

92. C. A. Burns, P. Abbamonte, E. D. Isaacs, P. M. Platzman, Plasmons in lithium ammonia. Physical Review Letters 83, 2390-2393 (1999). 
Acknowledgments: SEB and RSM thank Peter Vöhringer and PJ thanks Tomas Jungwirth for valuable discussions. All authors acknowledge the Helmholtz-Zentrum Berlin for allocation of synchrotron beam time at BESSY-II. OM, TM, KB, MC, AG, and PJ acknowledge the Czech Ministry of Education, Youth and Sports for generous allocation of supercomputer time at IT4I in Ostrava via the Large Infrastructures for Research, Experimental Development and Innovations project „IT4Innovations National Supercomputing Center - LM2015070.

Funding: PJ thanks for support to the European Regional Development Fund (project ChemBioDrug no. CZ.02.1.01/0.0/0.0/16_019/0000729). OM was supported by the grant Primus16/SCI/27/247019. SEB and RSM are supported by the US National Science Foundation (CHE-1665532). TM acknowledges fellowship from the University of Chemistry and Technology, Prague, KB from the Charles University, and both from the IMPRS Dresden. RSM acknowledges support from the States of Jersey Postgraduate Bursary. RS acknowledges the Deutsche Forschungsgemeinschaft for awarding an Emmy-Noether grant (No. SE 2253/3-1). BW acknowledges support from the Max-Planck-Gesellschaft. ST acknowledges support from the JSPS KAKENHI Grant No. JP18K14178. HA thanks the Egyptian Ministry of Higher Education and Ain Shams University for her PhD grant and the Egyptian Culture Office in Berlin for support. PEM acknowledges support of his YouTube popular science channel Thunderf00t.

Author contributions: TB, PEM, RSM, CS, BW, SEB, and PJ designed the experiment and together with RS, HA, DH, GW, and KB performed the measurements at the BESSY II synchrotron and ST assisted with data analysis. OM, TM, and PJ designed the computational 


\section{DIAAAS}

approach. TM, KB, MC, and AG performed the calculations. PJ, BW, and SEB wrote the manuscript with comments from all the authors.

Competing interests: Authors declare no competing interests.

Data and materials availability: All data is available in the main text or the supplementary materials or in a freely accessible repository Zenodo(65).

\section{Supplementary Materials:}

- Materials and Methods

- Supplementary Text

- $\quad$ Figs. S1 to S28

- References (66-92) 


\section{Science}

Submitted Manuscript: Confidential

\section{DIAAAS}

Fig. 1. Experimental PE spectra of alkali metal - liquid ammonia solutions at varying concentrations as obtained by synchrotron X-ray photoelectron spectroscopy in a refrigerated liquid microjet setup. (A) $\mathrm{Li}$ in $\mathrm{NH}_{3}$, (B) $\mathrm{Na}_{\text {in }} \mathrm{NH}_{3}$, and (C) $\mathrm{K}$ in $\mathrm{NH}_{3}$. The individual data points are color-coded to reflect the actual color of the solutions. The energy scales are with respect to the vacuum level. (D) Integrated peak areas from 0 to $6 \mathrm{eV}$ in (A) - red, (B) - purple, and (C) - yellow, as a function of the alkali metal concentration.



\section{Science}

Submitted Manuscript: Confidential

MIAAAS

Fig. 2. Ammoniated electron and dielectron simulated by ab initio molecular dynamics. We present radial electron density profiles calculated from the squares of the corresponding Wannier orbitals (green filled curves) and the center of excess charge - ammonia nitrogens radial distribution functions (blue curves). Normalization is such that the integrated excess electron density of the dielectron is twice that of the electron (the latter being arbitrarily set to peak at the value of 1). The dashed vertical lines denote the electron or dielectron radii of gyration. Insets: Snapshots of the squared Wannier orbitals with surrounding ammonia molecules in the AIMD simulation box. A. AIMD results for the ammoniated electron. B. AIMD results for the ammoniated dielectron.

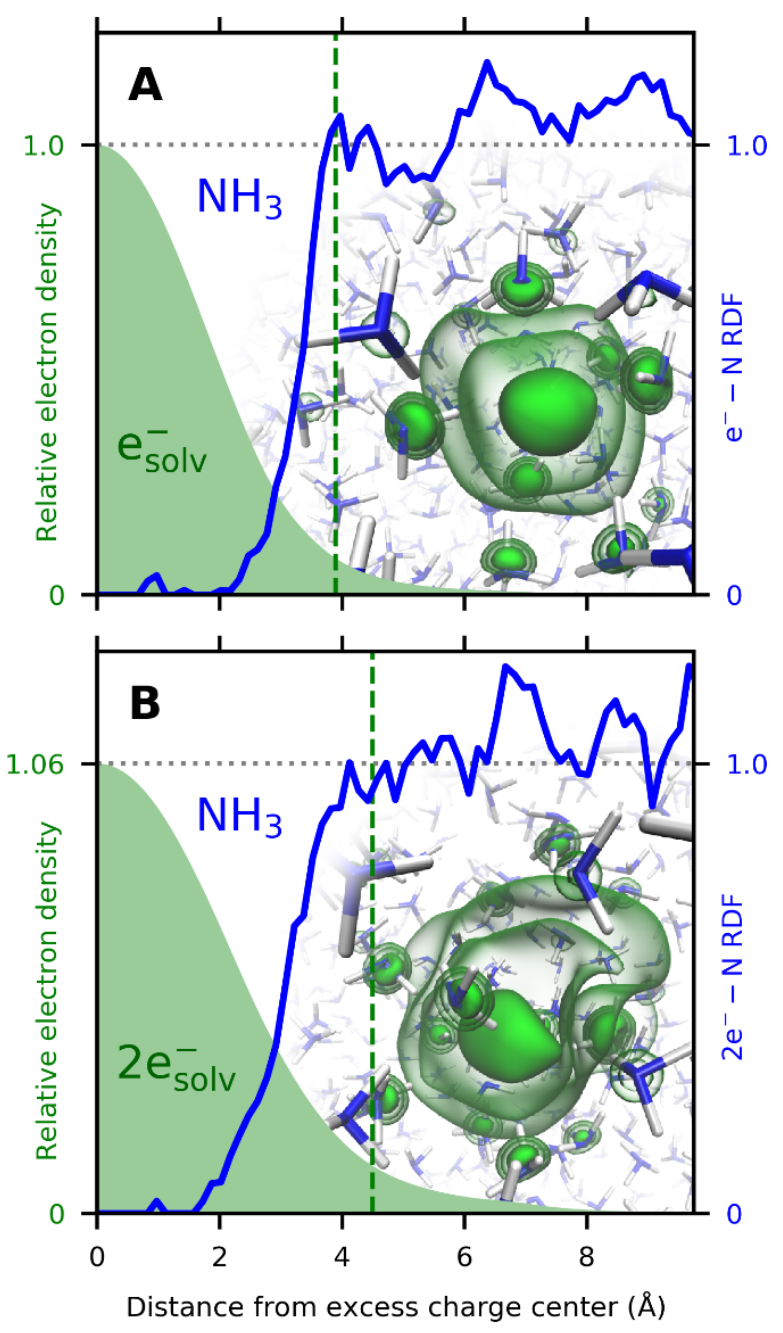




\section{Science}

Submitted Manuscript: Confidential

\section{DIAAAS}

Fig. 3. Simulated vertical detachment energies. Ammoniated electron (red) and dielectron

(purple) were modeled using solvation shells with 12 ammonia molecules carved out from

AIMD simulations and embedded in PCM. For comparison we depict the corresponding section of experimental PE spectra of the low-concentration lithium - ammonia solutions (from Figure 1A).

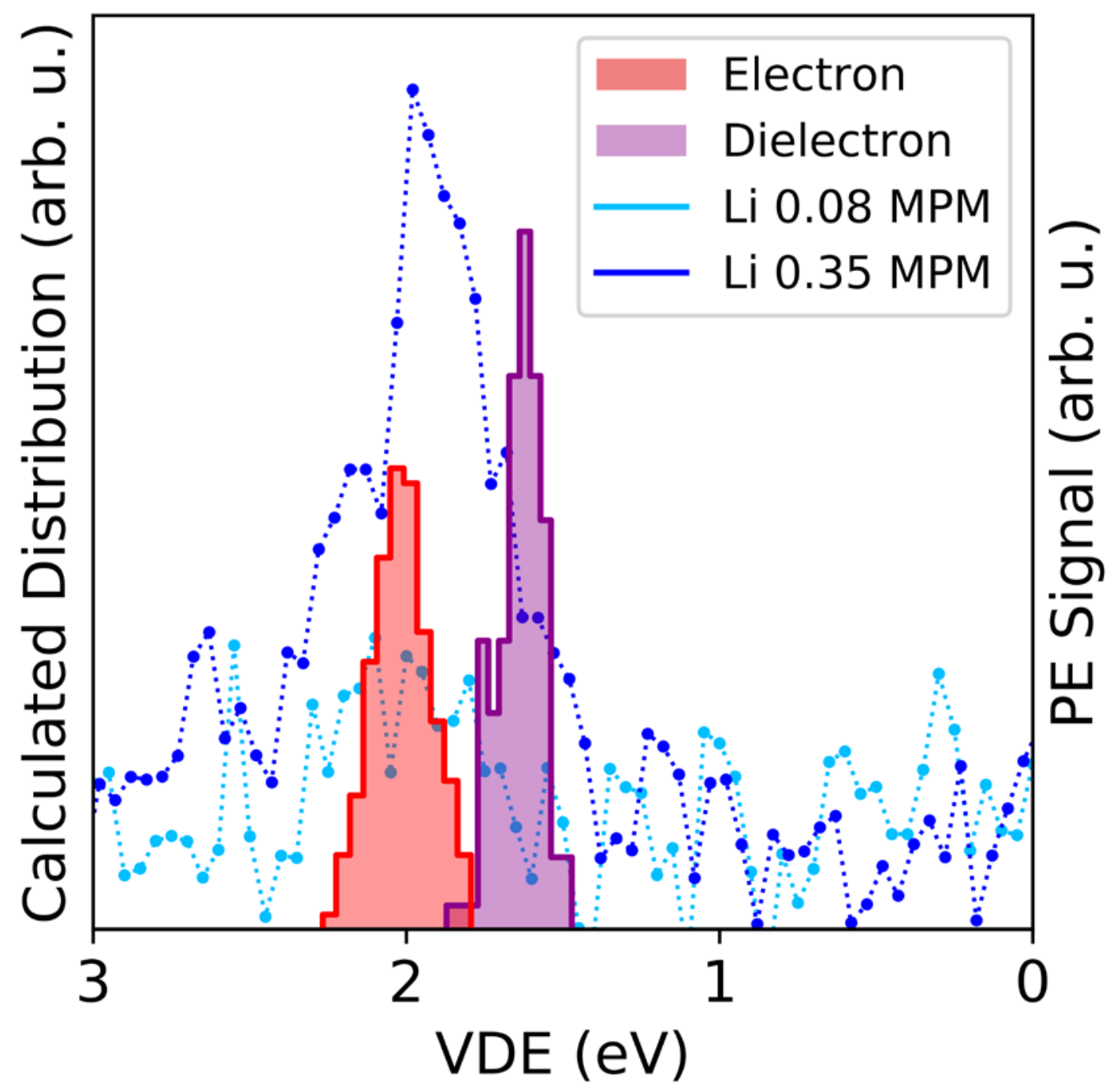




\section{DIAAAS}

Fig. 4. Analysis and fits of the photoelectron spectra in the electrolyte and metallic regimes. Note that the partial fits to the conduction band, plasmons, and localized (di)electrons are vertically offset for better visual clarity. A. Fit (relative root mean square error of $5.3 \%$ ) of the liquid $\mathrm{Na}-$ K alloy to a free electron gas model, comprised of, from low to high binding energy, a Fermiedge leading feature with characteristic parabolic shape of the conduction band with plasmon excitations as higher binding energy features and B. Fits (relative root mean square errors of 6.3, 6.8, 7.1, 8.9, and 32.2 \% for 9.7, 3.4, 0.97, 0.35, and 0.08 MPM, respectively) of $\mathrm{Li}-\mathrm{NH}_{3}$ data with a combination, in varying ratios, of a free electron gas model with plasmon bands for the fraction where the electron is delocalized and a single Gaussian to represent the localized (di)electron. C. Evolution of the liquid ammonia 3a1 peak upon increasing Li concentration. D. Concentration dependence of the effective electron mass from fits in A and B. E. Relative peak areas corresponding to the localized Gaussian, the conduction band, and the plasmon peaks in B. 

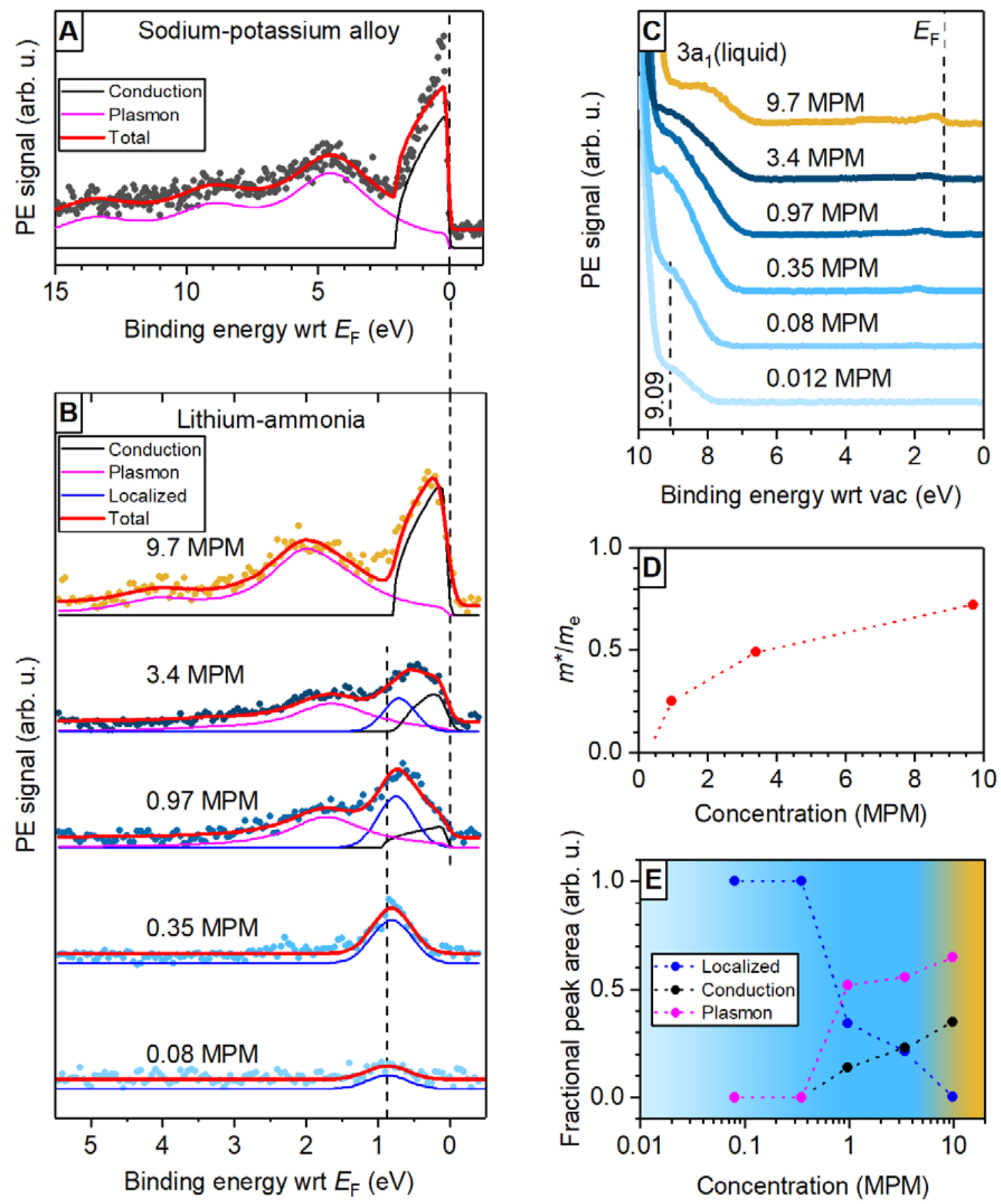


\section{Science \\ Submitted Manuscript: Confidential}

MIAAAS

Fig. 5. Picture emerging from band formation during the transformation from electrolyte (blue) to metallic solution (bronze/gold) upon increasing alkali metal concentration. The limiting photoelectron spectra are schematically represented at the top and bottom of the figure with axes showing binding energies with respect to the Fermi and vacuum level respectively. For a free electron gas (upper concentration limit), the width of the leading conduction band is expected to scale with electron number density with the plasmon bands, Ep, simultaneously moving out in spacing from the leading edge.



